\title{
Stop that glucose getting to heart!
}

\author{
Kshama Wechalekar, MBBS, DRM, DNB (Nuclear Medicine) ${ }^{\mathrm{a}}$ \\ a Department of Nuclear Medicine, Royal Brompton and Harefield Foundation Trust Hospital, \\ London, UK
}

Received Sep 7, 2018; accepted Sep 7, 2018

doi: 10.1007/s12350-018-1447-9

\begin{abstract}
Cardiac inflammation/infection imaging with 18F-FDG has been very useful in guiding patient management in conditions such as cardiac sarcoidosis, myocarditis, device infections, and prosthetic implant-related infections. Various protocols have been described to suppress normal myocardial glucose uptake. Certainly, there is need for standardization of protocols but adaptation of protocol to suit individual patient needs is also equally important. In the current article in this issue, Alexandra Clément et al have shown that a 7-day ketogenic diet can be used as an alternative method in mice models. This may work well in humans who are unable to fast.
\end{abstract}

Key Words: Sarcoid heart disease $\cdot$ inflammation $\cdot$ myocarditis $\cdot$ metabolic $\cdot$ PET

\section{See related article, pp. 612-618}

The beauty of cardiac inflammation imaging with 18F-FDG-PET comes with the responsibility of glucose uptake suppression in the most metabolically active organ-the myocardium. Versatile myocardium uses various substrates to maintain its vigorous function, glucose being the major one via insulin and GLUT-4 transporter. If starved of glucose, it switches to fatty acids and other substrates. Achieving this switch has been discussed in various metaanalyses ${ }^{1}$ and reviews ${ }^{2}$ and articles including the one in the current issue by Alexandra Clément et al titled $A$ one-week extension of a ketogenic diet provides a further decrease in myocardial $18 F-F D G$ uptake and a high detectability of myocarditis with FDG-PET. A 7-day ketogenic diet (high fat and protein but no carbohydrate) showed good suppression of glucose uptake in the mice hearts than pure 18 hour fasting. This is important information since it adds another possible option to various other existing protocols using combination of fast, dietary alteration, and

Reprint requests: Kshama Wechalekar, MBBS, DRM, DNB (Nuclear Medicine), Department of Nuclear Medicine, Royal Brompton and Harefield Foundation Trust Hospital, Sydney Street, London SW3 6NP, UK; k.wechalekar@rbht.nhs.uk

J Nucl Cardiol 2020;27:619-20.

$1071-3581 / \$ 34.00$

Copyright (C) 2018 American Society of Nuclear Cardiology. heparin. This new alternative gives hope to patients who are unable to fast for any longer than overnight. The only caveat being mice are in a cage feeding on carefully calculated diet with no personal choice!

High fat, high protein, and no/low carbohydrate diet, prolonged fasting, heparin, verapamil has been described individually and in combination for varied durations are described with varying success rates. We must not forget that our scan results have major implications on patient management so getting the basic metabolic preparation correct is of utmost importance. Moreover, need for a repeat scan adds to radiation burden and delay in management. Myocardial inflammation is always patchy in conditions such as sarcoidosis and myocarditis, which can be seen as areas of variable uptake in the myocardium. However when the myocardium shows homogeneously increase uptake, there is always a suspicion of failure of protocol, although in rare cases this could also be due to severe myocardial inflammation as shown in Figure 1. Going back to the patient with questions about their diet after the scan usually is helpful to solve the dilemma in addition to a good clinical history and multi-disciplinary discussion.

Achieving minimal counts in the myocardium compared to the blood pool is what we strive to achieve. This has not been an easy task in our experience of scanning over a thousand patients. It needed a cultural change on inpatient wards with education of staff members, patients, family members, and doctors. Empowering patients with do's, don'ts, menus and 


\section{Protocol Compliance}

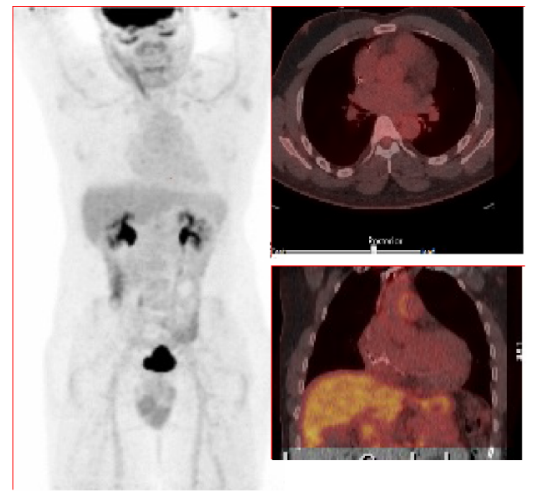

A

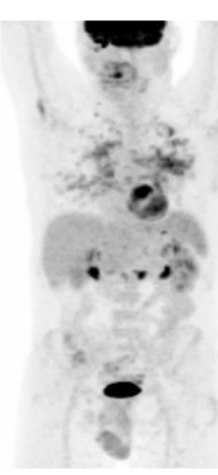

B
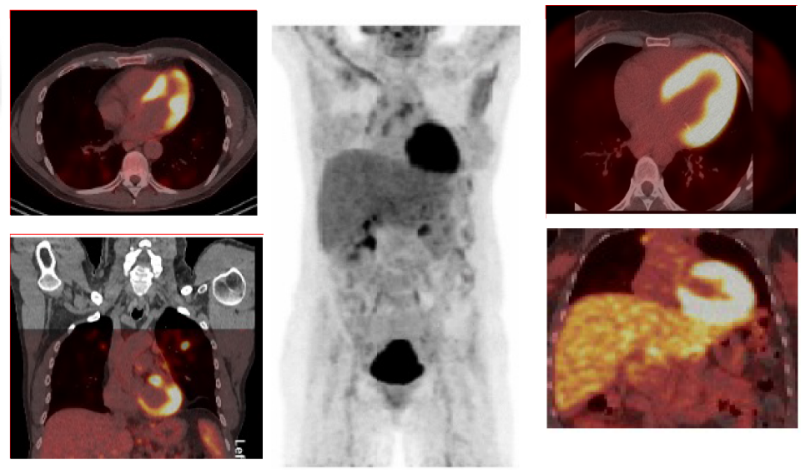

C

Figure 1. (A) preparation compliance-negative study with blood pool activity; (B) preparation compliance-positive study with patchy cardiac uptake and (C) preparation Failure with homogeneously increased uptake in the myocardium.

explaining 'why' has been essential. Detailed instructions from booking team and telephonic crosschecks have reduced our non-diagnostic scan rates to $1 \%$ in a sample dataset of 100 consecutive patients. ${ }^{3}$ Of course, we had to swallow a bitter pill of cancelling the exam on the day, on reporting breach of preparation. We use an 18-hour high fat, high protein, and no carbohydrate diet followed by an 18-hour fast protocol. Every institution can standardize own protocol taking advice from the guidelines. Current SNMMI/ASNC/SCCT guidelines recommend preparation with a diet rich in fat and devoid of carbohydrates for 12-24 hours prior to the scan, a 1218 hours fast, and/or the use of intravenous heparin approximately $15 \mathrm{~min}$ prior to $18 \mathrm{~F}-\mathrm{FDG}$ injection. ${ }^{4}$

Of course, one size does not fit all! The nuclear cardiology community is need of a simple, reliable bedside biomarker that can be checked prior to FDG injection such as serum FFA, serum ketone bodies or serum insulin to confirm adequacy of patient preparation. This will allow plurality of protocols to suit various needs of our patients. In this era of personalized medicine, we have to adapt to individual patient needs such as diabetics-more careful approach with monitoring glucose levels and adjusting the anti-diabetic medication/insulin accordingly, anticoagulated patients-no heparin and patients who can not tolerate fast-can now have an option of no carbohydrate diet for 7 days! Caution: Cage not included!!

\section{Disclosure}

Kshama Wechalekar has nothing to disclose.

\section{References}

1. Osborne M, Hulten E, Murthy V, Skali H, Taqueti V, Dorbala S, et al. Patient preparation for cardiac fluorine-18 fluorodeoxyglucose positron emission tomography imaging of inflammation. $\mathrm{J}$ Nucl Cardiol 2017;24:86-99. https://doi.org/10.1007/s12350-016-0502-7.

2. Tang R, Wang JT, Wang L, Le K, Huang Y, Hickey AJ, et al. Impact of patient preparation on the diagnostic performance of ${ }^{18} \mathrm{~F}$ FDG PET in cardiac sarcoidosis: A systematic review and metaanalysis. Clin Nucl Med 2015. https://doi.org/10.1097/RLU. 000000000001063

3. Bagi E, Garcia-Campos A, Hartigan W, Wechalekar K. Patient compliance with myocardium metabolic suppression protocol for 18F-FDG PET Sarcoid Scan OP 278 EANM 2017.

4. Dorbala S, Di Carli MF, Delbeke D, Abbara S, DePuey EG, Dilsizian V, et al. SNMMI/ASNC/SCCT guideline for cardiac SPECT/CT and PET/CT 1.0. J Nucl Med 2013;54:1485-507. 